\title{
Effets de l'âge d'immersion sur la compréhension initiale d'expressions modales épistémiques en français L2
}

\author{
Cyrille Granget ${ }^{1,1}$, Marie-Ange Dat ${ }^{1}$, Christine Cuet $^{1}$, Pascale El Haj ${ }^{2}$, Fatima Albochi ${ }^{1}$, et \\ Ashraf Allawama ${ }^{1}$ \\ ${ }^{1}$ Laboratoire de Linguistique de Nantes, UMR 6310, Université de Nantes \& CNRS, France. \\ ${ }^{2}$ Structures Formelles du Langage, UMR 7023, Université de Paris 8 \& CNRS, France.
}

\begin{abstract}
Résumé. Alors qu'il est établi qu'à l'âge adulte le locuteur précoce d'une langue seconde dépasse dans de nombreux tests de performance le locuteur tardif, une question majeure reste de savoir si dès le stade initial l'enfant dispose d'une forte capacité acquisitive ou d'une marge de progression plus large. Notre étude observe si dans les 15 premiers mois d'exposition au français, des locuteurs enfants, adolescents et adultes de l'arabe syrien (L1) comprennent la valeur d'épistémicité de différentes expressions modales. Cet effet de loupe sur la compréhension d'expressions modales épistémiques dans les stades initiaux par des apprenants d'âges différents montre des avantages comparatifs du groupe adulte sur les groupes adolescents et enfants.
\end{abstract}

\begin{abstract}
Whereas it is well known in second language acquisition that adults who started learning early have better scores in several performance tests than those starting later, a central issue is to know if children have advantages right from the beginning. Our study deals with this issue by looking at the understanding of epistemic modal expressions by children, adolescents et adult learners of French L2 during the first months of intensive exposure to French. We have shown that the well known advantages of early learners are not true for the epistemic domain right from the beginning. Adults have advantages over children and adolescents by understanding epistemic expressions at the initial states.
\end{abstract}

\section{Introduction}

L'idée communément admise selon laquelle les enfants ont un avantage comparatif par rapport aux adultes dans l'acquisition d'une L2 s'appuie essentiellement sur des études de performance au niveau du stade ultime de développement (Hyltenstam \& Abrahamsson, 2003 ; Abrahamsson \& Hyltenstam, 2009) ou sur l'étude de productions en L2 (Dimroth, 2008; Kihlstedt, 2015). Seule l'étude de synthèse de Krashen, Long \& Scarcella (1979) semble apporter quelques bémols en avançant qu'à durée d'exposition comparable, il existe

\footnotetext{
${ }^{1}$ Correspondante : cyrille.granget@univ-nantes.fr
} 
un avantage des adultes et des adolescents sur les enfants en matière de rythme de développement morphologique et syntaxique dans les stades initiaux. Afin de mieux comprendre les différences liées à l'âge dans l'acquisition d'une langue seconde, nous proposons d'étudier un aspect négligé de la recherche en acquisition des langues seconde, l'effet de l'âge sur la compréhension dans les stades initiaux. Les enfants vont en moyenne plus loin que les adultes mais sont-ils plus rapides qu'eux? L'objectif de notre étude est de comparer la compréhension d'expressions modales épistémiques en français L2 par 39 apprenants arabophones du français L2, répartis en trois groupes d'âge distincts $(6-10,11$ $17,18+$ ). Sachant des préférences selon l'âge pour des moyens lexicaux vs grammaticaux en production L2 (Dimroth, 2008; Kihlstedt, 2015; Schlyter, 2003), trois modalités d'encodage - et de placement- de la modalité épistémique ont été retenues: adverbes épistémiques en position médiale, verbes assertifs ou modaux épistémiques en position médiale et verbes lexicaux épistémiques en position initiale. La tâche de compréhension consiste à deviner à partir de deux affirmations épistémiquement distinctes où se cache un objet, une tâche devenue classique dans les études de la compréhension de la modalité en L1 (Byrnes \& Duff, 1989; Hirst \& Weil, 1982; Moore, Pure, \& Furrow, 1990; Noveck, Ho, \& Sera, 1996). Dans le cas présent, le volume d'exposition est contrôlé par une condition de durée de séjour en France inférieure à 15 mois et un questionnaire sociolinguistique.

Les comparaisons de performances d'adultes selon leur âge d'arrivée dans la communauté linguistique ne permettent pas de comprendre le déroulement du processus. On peut en effet imaginer deux scénarios explicatifs des limitations dans l'acquisition L2 (S1 et $\mathrm{S} 2$ ):

- S1 : la performance pour un fait de langue donné est une fonction du temps d'exposition les apprenants progressent lentement vers le stade ultime, mais la pente, et donc le résultat final, est différent pour les enfants et les adultes. Le début est le même, c'est vers la fin du processus que les adultes fossilisent.

- S2 : pour un fait de langue donné, le début du processus est différent selon l'âge de début d'acquisition : les adultes atteignent rapidement le point de fossilisation, tandis que les enfants sont plus lents mais dépassent le point de fossilisation « adulte » et présentent au final, en moyenne, de meilleures performances.

Un objectif de cette étude contrastive de la compréhension par des enfants, adolescents et adultes au stade initial est de préciser, pour les expressions modales épistémiques, quel scénario est le plus pertinent.

\section{Les effets de l'âge sur l'acquisition d'une L2, état des lieux}

Que l'âge de début d'acquisition soit corrélé à des niveaux plus élevés d'acquisition, qu'il joue un rôle contributif indépendant, en raison d'une certaine forme de contrainte maturationnelle, ou encore un rôle modéré dans un processus développemental multidimensionnel est l'une des questions les plus discutées dans les études sur le bilinguisme comme le rappelle Mitchell et Myles (2004 : 24) :

Do child and adult second language learners learn in essentially similar ways? Or, is there a critical age that divides younger and older learners, a moment when early learning mechanisms atrophy and are replaced or at least supplemented by other compensatory ways of learning?

Il existe de nombreux états des lieux récents des effets de l'âge sur l'acquisition d'une langue, lesquels comparent différents types de locuteurs, monolingues (L1) et bilingues (2L1), bilingues simultanés et successifs, ou bilingues avec langue A dominante et bilingues avec langue B dominante (Dimroth, 2009; Schmidt, 2014; Kupisch, Lein, Barton 
et al., 2014; Granena et Long, 2013). Même si la connaissance du développement linguistique en langue première permet d'appréhender des aspects du développement linguistique en langue seconde, l'état des lieux suivant porte sur les effets de l'âge sur l'acquisition d'une L2. Il compare ainsi l'acquisition d'une langue par des locuteurs âgés de 6 ans et plus et distingue trois groupes d'âge, enfants (6-10), adolescents (11-17) et adultes (18 et plus). Si l'âge de 10-11 ans est communément considéré comme une frontière séparant deux catégories de sujets sur la base de leurs performances à l'âge adulte (Meisel, 2011; Singleton et Ryan, 2004), la partition des locuteurs de onze ans et plus en deux catégories, ici adolescents et adultes, est pratiquée dans plusieurs études, avec des frontières d'âge variables (20 ans pour Byalistok et Hakuta, 1994; 15 ans pour Johnson et Newport, 1989 et Granena et Long, 2013; 16-17 ans pour Birdsong et Mollis, 2001). La composition de la catégorie adultes n'a guère été questionnée jusqu'à présent. C'est une classe d'âge hétérogène et la plupart des études mentionnées ci-dessus y incluent des participants dont l'âge maximum varie, de 29 ans (Granena et Long, 2013) à 39, 44 ou 47 ans (respectivement Johnson et Newport, 1989; Birdsong et Mollis, 2001; Abrahamsson et Hyltenstamm, 2009).

\subsection{Plus on est jeune, mieux c'est ? Le biais méthodologique de l'hypothèse de la période critique}

Fortement influencées par les résultats de Lenneberg (1967) auprès d'enfants sourds, de nombreuses études en langue seconde ont cherché à tester l'hypothèse selon laquelle la maturation progressive du cerveau de l'être humain s'accompagne d'un déclin de sa propension à atteindre, dans la langue à laquelle il est exposé, un stade comparable à celui affiché par des locuteurs de la L1. L'étude souvent citée de Johnson et Newport (1989) abonde en ce sens. Plus récente, l'étude de Abrahamsson et Hyltenstamm (2009) confirme aussi l'existence d'une période critique autour de l'âge de 11 ans. L'étude s'appuie sur des scores à un jugement de grammaticalité, pour partie oral, pour l'autre écrit, effectué auprès de locuteurs du suédois L1 et d'apprenants du suédois L2 jugés " natifs » par des locuteurs du suédois L1. Parmi eux, certains l'ont appris avant 11 ans et d'autres après. A l'oral, 58 $\%$ de ceux qui ont appris le suédois précocement ont des scores similaires aux natifs contre $40 \%$ de ceux qui l'ont appris tardivement. A l'écrit, $65 \%$ des apprenants précoces réalisent la tâche comme les natifs, contre $50 \%$ de ceux qui l'ont appris tardivement. Au niveau des temps de réaction, $94 \%$ des apprenants précoces réagissent aussi vite que les natifs contre $60 \%$ des apprenants tardifs. Autrement dit, les apprenants de L2, quel que soit leur âge de début d'acquisition, ont des représentations grammaticales distinctes de celles du groupe natif, quand bien même ils sont considérés comme des natifs sur la base de leur prononciation. Mais il existe une différence entre des apprenants précoces et tardifs en termes de représentation et de temps de réaction dans la tâche: plus l'apprentissage est tardif, plus l'intuition grammaticale est susceptible de s'altérer. Ces résultats et d'autres issus d'études similaires ont généralisé l'idée selon laquelle l'âge contraint et limite l'apprenant de langue seconde. Au niveau segmental et suprasegmental, l'étude de Hyltenstam et Abrahamsson (2003) auprès des 195 bilingues espagnol-suédois se considérant comme des bilingues confirme ces contraintes maturationnelles. Les participants sont arrivés en Suède entre l'âge de 1 et 47 ans. Pour des locuteurs du suédois L1, la plupart de ceux qui sont arrivés avant l'âge de 12 ans sont considérés comme des suédophones natifs sur la base de leur prononciation du suédois. Mais seule une minorité de ceux qui sont arrivés après cet âge sont considérés comme tels. Ici l'âge apparaît comme un facteur prédictif de la capacité à maîtriser le système phonologique et prosodique de la L2. Pour expliquer ces différences entre enfants et adultes, Newport (1990) avance que les jeunes enfants se révèlent paradoxalement plus compétents que les adultes dans 
l'acquisition d'une langue seconde car leurs capacités cognitives plus limitées les conduisent à appréhender des unités plus petites que ne le font les adultes qui analysent des unités complexes. Ce que l'auteur formule de façon synthétique par « less is more ».

Pour comprendre le succès et les limites de cette hypothèse de la période critique ou des contraintes maturationnelles, il importe de souligner la conception de l'acquisition et les principes méthodologiques qui la sous-tendent. Ces résultats sont en effet liés à des présupposés théoriques et des routines méthodologiques. Ainsi, cette hypothèse qui met en relation des performances de locuteurs adultes avec l'âge de première exposition à cette langue, est liée à une conception de l'acquisition d'une langue seconde comme un processus biologiquement déterminé et fondamentalement déficitaire par rapport à l'acquisition de la langue première (cf. Birdsong, 2005 pour un développement de cette idée). Le focus restreint sur un construit de connaissances en L2 observées à l'âge adulte et sur l'âge au moment de l'immigration dans la nouvelle communauté linguistique, montre que ceux qui ont été exposés à la langue dès leur enfance ont en moyenne des résultats meilleurs que ceux qui y ont été exposés seulement à l'âge adulte. Ces derniers ont toutefois été exposés bien moins longtemps à la langue et parmi eux, certains ont atteint ce stade quasi-natif qui sert de référence.

Les études qui valident la période critique montrent clairement le poids variable de l'âge de première exposition dans l'équation acquisitionnelle. Plus le sujet commence tôt l'acquisition d'une langue seconde, plus la probabilité est élevée que ses performances morphosyntaxiques en reconnaissance ou segmentales et suprasegmentales en production ressemblent à celles de locuteurs dont c'est la langue première. La plupart de ces études sont des études transversales qui considèrent implicitement que le processus acquisitionnel est au stade ultime, soit abouti, au moment de l'enregistrement. Ces études ne nous indiquent pas quel est l'effet de l'âge de première exposition sur la forme de la trajectoire acquisitionnelle, sur les pratiques réceptives et productives à différents âges, notamment au début du processus acquisitionnel, ni sur les usages spontanés, ni sur la compréhension du sens de l'énoncé ou du discours ni encore sur les moyens linguistiques déployés pour réaliser un acte de langage ou assurer la cohérence d'un discours, autant d'éléments dont la connaissance permettrait aussi de comprendre les trajectoires acquisitionnelles de locuteurs commençant leur acquisition à des âges distincts et dans des conditions souvent distinctes également.

\subsection{Quelques nuances (ou dans quelle mesure " plus on est vieux, mieux c'est »)}

Même si l'hypothèse de la période critique a connu un grand succès, sa portée a été maintes fois nuancée. Plusieurs études ont en effet montré que l'âge de première exposition n'est pas un facteur prédictif absolu mais relatif (Birdsong \& Mollis, 2001; Schmidt, 2014), qu'il constitue un indicateur non pas tant du stade ultime de développement que du type d'apprentissage mis en œuvre, implicite vs explicite (DeKeyser, 2013), ou qu'enfants et adultes suivent des étapes comparables dans l'appropriation des propriétés de la langue cible (Schimke et Dimroth, 2017). Plusieurs chercheurs dont Köpke (2004) plaident ainsi en faveur de l'hypothèse d'une phase sensible, et constatent que les apprenants adultes ont des difficultés à atteindre en langue cible un niveau proche des locuteurs natifs. L'âge n'est pas le seul facteur explicatif des performances mesurées et usages observés en L2, mais c'est un facteur prépondérant à une certaine période, lorsque l'individu est jeune. Birdsong et Mollis (2001) qui répliquent l'étude de Johnson et Newport (1989) auprès d'apprenants hispanophones de l'anglais L2, suggèrent que les performances des locuteurs adultes dépendent également de la proximité typologique des langues en contact et d'autres facteurs comme la motivation. D'autres études soulignent la difficulté d'isoler l'effet de l'âge de 
l'effet du transfert de la L1 dans l'étude des performances d'apprenants de langue seconde (Schmidt 2014). D'une façon générale, ces études montrent que plus l'âge de première exposition est élevé, plus il s'avère nécessaire de prendre en compte d'autres variables explicatives pour rendre compte des performances variables des individus adultes dans les tâches mises en œuvre.

Il est toutefois permis de penser que d'autres observables permettraient d'enrichir notre compréhension des effets de l'âge de commencement de l'acquisition. Peu d'études se sont par exemple intéressées à l'effet de l'âge sur le rythme d'acquisition, une dimension pourtant essentielle pour comprendre la forme de la trajectoire acquisitionnelle des enfants et des adultes. Synthétisant quelques études des années 70s, Krashen, Long et Scarcella (1979) concluent, à contre-courant des propositions de Lenneberg (1967), qu'à durée d'exposition constante et dans les stades initiaux du développement morphologique et syntaxique, les adultes vont plus vite que les enfants et les enfants plus âgés plus vite que les plus jeunes. Contrairement à l'hypothèse de la période critique, cette proposition n'a toutefois guère été testée dans les recherches ultérieures. Gaonac'h (2006) montre cependant que l'acquisition d'une L2 est plus rapide pour les adolescents et les adultes que pour les enfants, mais que ceux-ci n'atteignent pas le même niveau de maîtrise que les locuteurs plus jeunes. Cette conclusion montre que l'effet positif du jeune âge dans l'acquisition d'une langue seconde dépend bien des observables retenus, et en matière de rythme, les locuteurs plus âgés semblent afficher un avantage.

C'est également la conclusion de Dimroth et Haberzettl (2012) qui comparent trois jeunes apprenantes russophones de l'allemand L2, âgées de 8;7, 7;5 et 8;6 aux locuteurs de l'allemand L1. En écho à Newport (1990), les auteurs avancent que l'augmentation des capacités cognitives avec l'âge peut aussi faciliter le traitement de l'input en L2 et permettre une acquisition globalement plus rapide à durée d'exposition égale, ce que les auteures résument par deux principes « the older the better» et "more is more ». L'âge ne semble pas être le seul facteur susceptible d'expliquer l'avantage des jeunes enfants en L2, par rapport aux adultes L2 et aux enfants L1. Les capacités cognitives accrues de l'enfant et de l'adolescent, conjuguées à une capacité croissante d'abstraction, à une expérience linguistique antérieure et à une exposition régulière à la langue cible, contrairement à beaucoup d'adultes, sont susceptibles d'accroître leur réceptivité aux propriétés distributionnelles dans l'input, de permettre l'inférence de règles de fonctionnement à partir d'un ensemble plus restreint de verbes. Ce type d'étude, par une méthodologie différente (étude longitudinale de cas et productions spontanées), montre que la focalisation sur la dimension processuelle de l'acquisition permet aussi d'enrichir la discussion autour des effets de l'âge. Dimroth (2008) présente une étude longitudinale de l'appropriation de la finitude et de la négation par deux sœurs, apprenantes russophones de l'allemand L2, une enfant et une adolescente respectivement âgées de 8 ans et 7 mois et 14 ans et 2 mois au moment du premier enregistrement, pendant les six premiers mois de leur séjour en Allemagne, soit 24 enregistrements d'une heure environ. Les usages de l'accord et de la négation au fil des semaines sont comparés à ceux d'adultes L2 publiés dans des études antérieures. L'auteure montre que le développement de la finitude et de la négation en allemand L2 chez l'enfant est plus rapide que celui observé chez l'adolescente et il ne suit pas les mêmes étapes que celles habituellement observées chez les adultes et chez l'adolescente de l'étude. Ces résultats ne sont toutefois pas confirmés avec un échantillon élargi et une tâche d'imitation d'énoncés qui montrent que les adultes et les enfants suivent finalement les mêmes étapes dans l'acquisition de la finitude et de la négation en allemand L2 (Schimke et Dimroth, 2017). Mais ces résultats en termes de rythme et d'ordre acquisitionnel ont le mérite d'enrichir et nuancer le débat autour du facteur âge.

En résumé, le débat autour du rôle de l'âge dans l'acquisition d'une langue seconde a pâti d'une conception manichéenne de la question : l'âge a été envisagé comme un 
déterminant principal, les groupes comme des classes discrètes de pratiques réceptives ou productives, linguistiques ou métalinguistiques, et les performances à l'âge adulte comme un critère central d'évaluation de l'acquisition. Or l'âge n'est pas le seul facteur en jeu, d'autres facteurs permettent d'expliquer la variété des performances adultes. De la même façon, la performance en prononciation ou à un jugement de grammaticalité surdéterminent les conclusions en faveur du jeune âge alors que l'étude du rythme, des stades acquisitionnels ou d'autres dimensions du développement linguistique permet de saisir les multiples effets de l'âge en montrant tantôt un avantage des locuteurs plus âgés, tantôt des différences qualitatives, voire des similarités de parcours entre adultes et enfants.

Il ressort ainsi de cette synthèse que de nombreuses propriétés des langues ou dimensions du processus restent à mettre en relation avec l'âge de première exposition. Par exemple, peu d'études se sont penchées sur les effets de l'âge sur la compréhension en L2, notamment dans les stades initiaux, a fortiori sur la compréhension d'expressions modales épistémiques. Comme il existe des raisons de penser que l'échelle d'acquisition des expressions épistémiques en L1 permet d'anticiper si le développement d'expressions épistémiques en L2 est dépendant de l'âge ou de la quantité d'input, le développement des expressions épistémiques, en L1 et L2, en production et en compréhension, sera l'objet de la partie suivante.

\section{L'acquisition des expressions modales épistémiques en français}

Selon Ågren et al. (2014), les structures qui se développent précocement en L1 auront tendance à être acquises en L2 par les locuteurs les plus jeunes, mais difficilement par les adultes. En revanche, le développement des structures qui apparaissent tardivement en L1 est fonction non pas de l'âge mais de la quantité et la qualité d'input auquel sont exposés les locuteurs. C'est pourquoi il est intéressant d'étudier le développement en L1 des expressions épistémiques pour appréhender leur développement en L2.

\subsection{En L1, production et compréhension tardive des expressions épistémiques}

Les recherches sur le développement des expressions épistémiques en français L1 ont montré que leur production et leur compréhension est un phénomène tardif par rapport à d'autres phénomènes comme le développement de la finitude ou de la détermination. D'une manière générale, les expressions modales épistémiques apparaissent dans le discours oral spontané de l'enfant en langue première après les expressions déontiques et dynamiques. Stephany (1995) précise que les énoncés modalisés épistémiques sont rares jusqu'à 3-5 ans, mais des verbes ou adverbes épistémiques peuvent précéder ces modalisations. Toutefois les expressions épistémiques sont plus précoces en français qu'en anglais, ce qui pourrait être lié à l'usage fréquent de marqueurs épistémiques lexicaux en français et suggère donc une influence du mode d'encodage. En coréen par exemple, où l'expression épistémique est grammaticalisée, le marquage est observé vers 3 ans (Choi, 2005).

Dans une étude longitudinale de plus de trois ans, Bassano (1996) observe le développement d'expressions épistémiques dans le discours d'un enfant francophone. Chronologiquement, les expressions les plus précoces et les plus fréquentes sont celles de l'ignorance, puis les modalisations disassertives, à commencer par l'évaluation de la possibilité à $2 ; 7$ ans, les énoncés de croyance à $2 ; 9$ alors que l'affirmation de la probabilité apparaît tardivement à $3 ; 3$. Les jugements hypothétiques et interrogatifs apparaissent en dernier. Le tableau 1 ci-dessous présente les expressions utilisées par l'enfant. 
Tableau 1. Développement des expressions épistémiques en français L1 (Bassano, 1996)

\begin{tabular}{|l|l|l|l|}
\hline Âge & $\mathbf{2}-\mathbf{2 , 6}$ & $\mathbf{2 , 6} \mathbf{- 3 , 0}$ & $\mathbf{3 , 0} \mathbf{- 3 , 6}$ \\
\hline & je sais pas & $\begin{array}{l}\text { je sais (que) } \\
\text { je crois que }\end{array}$ & bien sûr \\
vrai, faux, sûr & $\begin{array}{l}\text { peut-être } \\
\text { conditionnel } \\
\text { je sais pas si }\end{array}$ & $/$ wwa/ (rare) \\
\hline
\end{tabular}

D'autres études ont montré que d'une part le développement de la compréhension des expressions épistémiques est fonction du type d'opérations modales qu'elles impliquent. D'autre part, la modalité épistémique est liée au développement cognitif de l'enfant, d'une perspective auto-centrée vers une prise en compte de la perspective de l'autre, pouvant se poursuivre jusqu'à 11 ans. Les modalisations assertives qui renforcent la force assertive de la proposition et expriment la certitude comme je sais que, je suis sûr sont comprises à partir de 4 ans. En revanche, les modalisations disassertives sont plus difficiles à comprendre à cet âge : des expressions comme peut-être, je ne suis pas sûr, je crois, je sais si ne sont pas complètement comprises et dissociées d'expressions de la certitude, jusqu'à l'âge de 7 ans pour certains d'entre eux (Bassano, Hickmann et Champaud, 1992; Bassano et Mendès-Maillochon, 1994). La modalité assertive je sais est comprise avant l'expression de l'ignorance je sais pas et la modulation du jugement je crois (Bassano 1985). On observe ici nettement un phénomène répertorié dans d'autres études sous le terme d'asymétries production/ compréhension (cf. Ünal et Papafragou, 2016 pour un état des lieux). A un âge donné, les enfants produisent des expressions épistémiques dont ils ne comprennent le sens que plus tard.

Cet état des lieux des recherches sur le développement des expressions épistémiques en L1 nous permet d'avancer que les expressions épistémiques font partie des structures d'apparition tardive si l'on s'en tient aux critères de Ågren et al., 2014, ce qui impliquerait un usage en L2 d'autant plus probable et approprié que le sujet est exposé à une quantité importante et variée d'input. L'âge ne devrait pas constituer dans ce cas précis un facteur prédictif. Aussi devrait-on observer une corrélation entre la durée d'exposition intensive au français de l'apprenant et sa compréhension des expressions épistémiques.

\subsection{En français $L 2$, quelques expressions épistémiques en production}

En français L2, le développement des expressions épistémiques a été étudié de façon longitudinale pendant trois ans auprès de trois apprenants de langue première arabe marocain (Véronique, 1995; Véronique, Stoffel, \& Giacomi, 2000). Pour exprimer la modalité épistémique, les auteurs relèvent dans les discours de Zahra, Abdessamad et Abdelmalek sur la période étudiée trois expressions verbales, les verbes cognitifs je krwa en (1) et (2), je sais pas en (2) et (3) et la locution verbale ça dépend, invariable dans le discours de l'apprenant.

(1) Abdelmalek, cycle 3

E : et là tu vas repartir à Nice aujourd'hui ? 
A : ouais euh je pars à cinq heures je /krwa/

(2) Abdessamad cycle 1 jeu de rôle au guichet d'une banque, transfert d'argent

E : oui, ben, il faut donner plus alors

A : ça dépendre onze pour cent neuf pour cent je/se/pas

(3) Zahra, cycle 1, conversation

c : ouais c'est le maître qui travaille

$\mathrm{Z}:$ oui

$\mathrm{c}:$ ouais d'accord et depuis combien de temps?

$\mathrm{Z}: \mathbf{j e} / \mathrm{krwa} /$ un mois je/se/pas

Les locuteurs utilisent les expressions épistémiques en position initiale ou finale d'énoncé. Stoffel et Véronique rapportent aussi le recours à des moyens kinésiques et prosodiques d'expression de la modalisation tels les mimiques, les rires, les pauses, qui, dans le contexte de l'énonciation, constituent des indices à partir desquels l'interlocuteur infère un sens modal. Ces indices linguistiques ne sont toutefois pas analysés systématiquement dans le cadre de l'article.

Ce qui est frappant à la lecture de ces études, c'est que d'une part, les expressions épistémiques utilisées par ces deux adultes, je sais pas et je crois sont aussi celles qui apparaissent en premier chez les enfants. D'autre part, par rapport aux productions d'enfants et aux moyens employés en L1 par les adultes, peu de moyens verbaux épistémiques sont employés par les apprenants de L2. A cet âge de maturité cognitive, l'hypothèse d'un obstacle cognitif est écartée. Une question qui se pose est donc de savoir si, à défaut de les employer, les apprenants comprennent les autres expressions épistémiques qui apparaissent avant 4 ans dans le discours de l'enfant, comme peut-être, bien sûr, je sais que, doit ou je sais pas si. Comme le souligne Mifka-Profozic (2017), il existe ici une véritable lacune dans la recherche: la compréhension des expressions modales n'a pas encore été étudiée en L2. Notre étude contribue à combler ce vide en apportant des éléments de réponse à cette question puisqu'elle observe si, dans leur acquisition initiale du français langue seconde, des enfants, adolescents et adultes attribuent une valeur d'épistémicité à ces expressions employées, pour la plupart, par un enfant entre 2 ans et 3 ans et 6 mois en français L1. Si l'hypothèse de Krashen, Scarcella et Long (1979) est pertinente, les adultes devraient avoir un avantage par rapport aux enfants, notamment en raison de leur maturité cognitive.

\section{La méthodologie}

\subsection{Participants}

Dans une étude sur les effets de l'âge dans les stades initiaux, les participants doivent constituer des groupes hétérogènes du point de vue de l'âge de première exposition à la L2 mais homogènes en termes notamment de durée d'exposition à la langue seconde et de langue première afin de pouvoir neutraliser dans l'interprétation des résultats l'effet d'un éventuel transfert.

\subsection{1 Âge}

39 apprenants du français langue seconde ont participé à cette tâche de compréhension des expressions épistémiques : 12 enfants âgés de 6 à 10 ans au moment de la première exposition intensive au français, 12 adolescents âgés de 11 à 15 ans et 15 adultes âgés de 18 
à 49 ans. L'hétérogénéité d'âge est plus importante dans ce dernier groupe. Dans les études de l'effet de l'âge, elle est toutefois habituelle (cf. partie 1) et n'est pas considérée comme un biais dans l'interprétation des résultats. La moyenne d'âge de première exposition intensive pour les adultes est de 27 ans, 13 ans pour le groupe adolescents et 8;5 ans pour le groupe enfants (cf. Tableau 2).

\subsubsection{Durée d'exposition intensive au français}

Tous les participants résident en France et sont exposés au français depuis plus de deux mois et moins de 15 mois (cf. Tableau 2). Nous considérons sur la base de cette durée qu'ils se situent tous dans une phase initiale d'acquisition de la langue seconde. Toutefois nous avons cherché au moyen d'un questionnaire sociolinguistique à établir une mesure plus précise de leur expérience individuelle d'exposition au français. La littérature récente sur l'effet de l'âge a en effet souligné l'importance de distinguer la durée de séjour dans la communauté linguistique ou durée de résidence et le volume d'exposition à la langue cible (Schmidt, 2014; Ågren et al., 2014). Cette dernière donnée constitue une mesure des données entrantes plus pertinente que la précédente dans la mesure où le séjour dans une communauté linguistique, même prolongé, n'implique pas des contacts langagiers avec ses membres et donc un apprentissage. Dans le questionnaire sociolinguistique, une version pour les adultes et une pour les enfants et adolescents (cf. annexes 1.1 et 1.2), nous avons demandé à chaque participant son âge, les langues parlées à la maison depuis sa naissance, son parcours scolaire, les langues étrangères qu'il a apprises et la durée d'apprentissage, sa date d'arrivée en France, s'il suit ou a suivi des cours de français, en présentiel ou en ligne, ou la classe qu'il fréquente, les langues qu'il parle au quotidien en dehors de ses cours de français, la fréquence des contacts en français, la part du contenu qu'il estime comprendre en regardant un film en anglais et en français, etc. Des questions non prévues ont aussi surgi face à certaines situations individuelles. Sur la base de ces réponses, il est apparu que le volume d'exposition reste difficilement objectivable et comparable dans la mesure où le chercheur n'a souvent accès qu'à une représentation de ce volume, à travers le témoignage somme toute limité du locuteur ou de ses parents ou enseignants dans le cas des enfants. Pour les élèves scolarisés, il est par exemple impossible d'évaluer au moyen d'un questionnaire le volume des échanges en français en classe et en dehors. C'est pourquoi nous avons opté pour une mesure qui tente d'évaluer en mois la période d'exposition intensive au français. Elle correspond à une durée de séjour en France corrigée, de laquelle est soustraite le temps durant lequel la personne interrogée indique ne pas avoir entendu parler la langue. La durée moyenne d'exposition intensive au français des adultes est ainsi de presque 7 mois, et de 5,2 et 5,5 mois pour respectivement les adolescents et les enfants. Même si les enfants et adolescents sont en moyenne moins exposés au français que les adultes, ils le sont plus régulièrement du fait qu'ils sont scolarisés.

Tableau 2. Âge moyen de première exposition intensive et durée moyenne d'exposition au français pour chaque groupe.

\begin{tabular}{|l|l|l|l|}
\hline & $\begin{array}{l}\text { Adultes } \\
\mathrm{n}=15\end{array}$ & $\begin{array}{l}\text { Adolescents } \\
\mathrm{n}=12\end{array}$ & $\begin{array}{l}\text { Enfants } \\
\mathrm{n}=12\end{array}$ \\
\hline $\begin{array}{l}\text { Âge moyen de 1 } \\
\text { exposition intensive }\end{array}$ & $\begin{array}{l}27 \\
(18-49)\end{array}$ & $\begin{array}{l}13 \\
(11-15)\end{array}$ & $\begin{array}{l}8,5 \\
(6-10)\end{array}$ \\
\hline $\begin{array}{l}\text { Durée moyenne } \\
\text { d'exposition }\end{array}$ & $\begin{array}{l}6,95 \\
(2-14)\end{array}$ & $\begin{array}{l}5,2 \\
(2,5-11)\end{array}$ & $\begin{array}{l}5,5 \\
(2,5-15)\end{array}$ \\
\hline
\end{tabular}




\begin{tabular}{|l|l|l|l|}
\hline intensive & & & \\
\hline
\end{tabular}

\subsection{3 $L 1$ arabe syrien}

Tous les participants à notre étude sont nés en Syrie et y ont été socialisés et scolarisés en arabe syrien, y compris à l'université pour ceux qui l'ont fréquentée. Le choix de participants syriens est motivé par des raisons scientifiques et historiques. Une première raison scientifique est qu'à ce jour les seules études de production d'expressions modales en français L2 ont été conduites auprès d'apprenants arabophones (L1 arabe marocain). Le choix de participants arabophones pour l'étude de la compréhension d'expressions modales en français L2 présente ainsi l'avantage non négligeable que les données de compréhension sont comparables avec les données de production recueillies dans ces précédentes études. Un autre motif scientifique est que les participants doivent se situer à un stade initial défini par une durée de séjour inférieure à 15 mois et ne pas avoir été exposés intensivement au français antérieurement. Notre choix de participants s'est donc immédiatement porté sur des personnes ayant récemment immigré en France. Sachant que le début du recueil de données en 2016 se situe dans une période historique d'installation en France de personnes syriennes, peu ou pas exposées au français dans leur scolarité antérieure, nous avons ciblé les locutrices et locuteurs de l'arabe syrien pour notre étude.

En arabe syrien, l'assertion simple qui localise un objet dans un lieu donné est un énoncé averbal, comme en (4)

l-bonbon be-l-Selbe l-hamra
le bonbon dans la boîte rouge
le bonbon est dans la boîte rouge

Il existe différents moyens linguistiques de modaliser l'assertion. L'usage d'une proposition initiale avec un verbe cognitif et un subordonnant, baศref ?enno (je sais que), be-Ste?ed (je crois que), ma baSref iza (je sais pas si), comme en (5), permet d'exprimer de manière propositionnelle le degré de certitude avec lequel le locuteur envisage la réalisation du contenu de l'énoncé.

$$
\begin{array}{ll}
\text { bałref Penno / be-\{tePed / ma baSref iza } & \text { l-bonbon be-l-\{elbe l-hamra } \\
\text { je sais que/ je crois que / je sais pas si } & \text { le bonbon dans la boîte rouge } \\
\text { Je sais que/ je crois que/ je sais pas si le bonbon est dans la boîte rouge }
\end{array}
$$

L'épistémicité peut aussi s'exprimer par l'emploi dans la proposition locative d'adverbes comme Pakid (bien sûr), ?al Paghlab (probablement) ou yemken (peut-être), en position médiale, finale ou initiale comme en (6a-c)
(6)
a.
1-bonbon,
huwwe
le bonbon
$i l$
2akid / 2al ?aghlab/ yemken be-1-Yelbe 1-hamra
rouge
bien sûr/probablement/peut-être dans la boîte
Le bonbon, il est bien sûr/probablement/ peut-être dans la boîte rouge
$b$.
1-bonbon, be-1-Selbe 1-hamra huwwe Pakid// Pal Paghlab/ yemken
c. $\quad$ 2akid / Pal Taghlab/ yemken 1-bonbon huwwe be-l-Selbe 1-ḥamra

D'autres adverbes comme bjuz et rubbama expriment aussi un degré d'épistémicité équivalent à yemken. Enfin, des moyens plus grammaticalisés comme yemken ykun (peut être) ou le:zem ykun (doit être) permettent comme en (7a-c), d'exprimer différents degrés d'épistémicité en position initiale, médiale, ou finale d'énoncé.

$\begin{array}{llll}\text { (7) a. } & \text { l-bonbon } & \text { yemken ykun /le:zem ykun be-l-Selbe z-zar?a } \\ & \text { le bonbon } & \text { peut être / doit être } & \text { dans la boîte bleue } \\ \text { b. } & \text { yemken ykun /le:zem ykun l-bonbon } & \text { be-l-Selbe z-zar?a } \\ \text { c. } & \text { 1-bonbon be-l-Selbe z-zar?a yemken ykun / le:zem ykun }\end{array}$

yemken est la forme du verbe imperfectif yemken (être capable) au singulier masculin de $3^{\text {ème }}$ personne. C'est un mot polysémique avec un statut syntaxique hybride (Vanhove, 
Miller et Caubet, 2009). Sur le plan sémantique, cette forme verbale figée exprime des valeurs déontiques et épistémiques. Sur le plan syntaxique, il peut être considéré comme un adverbe lorsqu'il est employé après le verbe fléchi, en début ou fin d'énoncé, comme auxiliaire modal avec ykun, forme imperfective du verbe être, voire comme particule modale, avant le verbe fléchi dans l'énoncé verbal. le :zem acquière sa valeur d'épistémicité en combinaison avec l'auxiliaire ykun. Les auxiliaires modaux yemken ykun et le:zem ykun témoignent d'une flexibilité syntaxique plus grande qu'en français.

La modalité épistémique s'exprime donc en arabe syrien au moyen de propositions cognitives initiales, d'adverbes et verbes modaux qui non seulement encodent différents degrés d'épistémicité mais aussi occupent dans l'énoncé des positions syntaxiques variées : initiales, médiales ou finales. Dans la mesure où l'épistémicité s'exprime en français au moyen de catégories linguistiques semblables et dans des positions syntaxiques attendues de locuteurs arabophones, on ne peut écarter un avantage par rapport à des locuteurs d'une langue première agglutinante dans laquelle l'épistémicité s'exprimerait au moyen de suffixes verbaux (japonais, turc), mais ce n'est pas ici notre propos. Les expressions modales du français sont opaques pour le locuteur de l'arabe, qu'elles soient lexicales ou grammaticales, et la tâche de l'apprenant arabophone en compréhension consiste à identifier les lexèmes ou morphèmes épistémiques et à leur attribuer une valeur d'épistémicité. Sauf à considérer que la transférabilité d'une langue est fonction de l'âge, les effets de transfert sont néanmoins neutralisés dans cette étude par le fait que tous les participants sont des locuteurs de l'arabe syrien L1.

\subsubsection{Autre}

Les enfants, adolescents et jeunes adultes arrivés en France en 2016 et 2017 ont connu des interruptions dans leur scolarité ou leurs études du fait de la guerre et des différentes étapes de leur exil. Plusieurs d'entre eux ont vécu dans des camps de réfugiés, dans des pays arabophones limitrophes (Jordanie, Liban) ou en Turquie, où ils ont poursuivi ou interrompu leur scolarité, ou encore travaillé pour les plus âgés. C'est ainsi que certains participants déclarent parler ou comprendre le turc ou soulignent bien maîtriser l'anglais pour ceux qui l'ont appris en Jordanie ou au Liban. Même si nous n'avons pas considéré $a$ priori que ces connaissances linguistiques puissent influencer la compréhension du français dans les stades initiaux, cette hypothèse n'est pas tout à fait exclue.

\subsection{Matériel}

Pour tester la compréhension d'expressions épistémiques, nous avons répliqué en français L2 le protocole initialement proposé par Hirst \& Weil (1982) pour l'anglais L1 puis répliqué par Byrnes \& Duff (1989), Moore, Pure, \& Furrow (1990) et Noveck, Ho, \& Sera (1996). La tâche consiste, pour chaque participant, à entendre deux affirmations localisant un bonbon dans une boite rouge et dans une boite bleue placées devant le participant, par exemple $(8 \mathrm{a})$ et $(8 \mathrm{~b})$, et à indiquer à l'enquêteur où se trouve le bonbon.

(8)

a. Le bonbon, il est bien sûr dans la boîte rouge

b. Le bonbon, il est peut-être dans la boîte bleue

Chaque affirmation comprend une expression épistémique et chaque paire d'affirmations contraste deux expressions, ci-dessus adverbiales, épistémiquement distinctes, par exemple bien sûr vs peut-être. Il est donc attendu que le participant qui comprend la différence de valeur d'épistémicité entre les deux expressions localise correctement le bonbon, dans la boite rouge dans le cas ci-dessus. Les affirmations préenregistrées sont présentées oralement à l'apprenant les unes après les autres. Pour chaque 
localisation correcte, le participant se voit attribuer un score de 1 et un score de 0 pour chaque réponse incorrecte.

Les participants sont exposés à 3 séries de 12 affirmations (cf. Tableau 3). Dans chaque série, chaque paire d'expressions épistémiques est répétée 4 fois afin de s'assurer de la robustesse de la compréhension du contraste épistémique.

Tableau 3. Paires testées en compréhension

\begin{tabular}{|l|l|l|}
\hline Série A (adverbe) & Série B (verbe modal) & Série C (verbe cognitif) \\
\hline bien sûr/ probablement & est/ doit être & je sais que / je crois que \\
\hline bien sûr/ peut-être & est/ peut être* & je sais que/ je sais pas si \\
\hline probablement/ peut-être & doit être / peut être & je crois que / je sais pas si \\
\hline
\end{tabular}

* la liaison n'est pas effectuée afin de distinguer le verbe modal peut + être de l'adverbe peut-être.

Le choix des séries découle de deux principes. D'une part, les enfants et les adultes sont différemment sensibles au type de moyens linguistiques, lexicaux et grammaticaux, servant à encoder des concepts comme le temps et donc potentiellement la modalité (Schlyter, 2003 ; Van Patten, 2004) ainsi qu'au positionnement de ces moyens, en position initiale, médiane ou finale d'énoncé. Selon ces études, les moyens lexicaux sont préférés en production par les adultes et les moyens grammaticaux par les enfants et pour les adultes, il est plus facile de saisir des moyens linguistiques situés en position finale ou initiale d'énoncés que des éléments intégrés à l'énoncé. Dans la série A, ce sont des adverbes, moyens lexicaux positionnés au milieu d'énoncé, qui sont utilisés. Dans la série $\mathrm{B}$, des verbes modaux, moyens grammaticalisés positionnés en milieu d'énoncé et dans la série $\mathrm{C}$ des verbes cognitifs, moyens lexicaux, situés en début d'énoncé. Selon l'hypothèse précédente, les apprenants adultes devraient accéder plus facilement au sens des expressions épistémiques de la série $\mathrm{C}$, puis de la série $\mathrm{A}$ et enfin de la série $\mathrm{B}$. Pour les enfants, ce sont les moyens grammaticalisés (série B) qui sont plus accessibles que les moyens lexicaux (série A et C). D'autre part, les adultes comme les enfants sont aussi sensibles à la fréquence et à la saillance de ces expressions épistémiques dans les données environnantes, ce qui nous a conduit à privilégier la compréhension des premières expressions épistémiques utilisées par l'enfant en français L1. Ces expressions, d'après Bassano (1996), figurent en gras dans le tableau 3 ci-dessus. L'observation de ces deux principes garantit une adaptation du protocole à des apprenants de langue seconde d'âge différent et au français.

L'échelle d'épistémicité a été par ailleurs vérifiée auprès de 30 locuteurs du français L1 au moyen d'un jugement d'épistémicité dans lequel les participants, étudiants en $4{ }^{\text {ème }}$ année d'université, ont lu 9 paires d'énoncés modalisés localisant un objet dans une boîte bleue et dans une boîte rouge. Concernant les verbes modaux et cognitifs, tous les locuteurs francophones valident les échelles d'épistémicité est $>$ doit être $>$ peut être et je sais que $>$ je crois que > je ne sais pas si, > signifiant "a une valeur d'épistémicité plus forte que". Concernant les adverbes, la supériorité épistémique de bien sûr par rapport à probablement et peut-être est également unanimement établie par l'ensemble des participants francophones L1. Seulement 24 étudiants sur 30 considèrent en revanche que probablement a une valeur d'épistémicité plus importante que peut-être, trois d'entre eux ne partagent pas cet avis et trois ne savent pas.

Globalement, ce test préliminaire a montré que, pour des locuteurs précoces du français L1, les expressions modales retenues présentent des valeurs épistémiques distinctes et contrastées. Il aurait été possible d'informatiser le protocole. Il a toutefois été décidé dans 
cette étude de maintenir une passation avec les boîtes comme cela a été pratiqué dans les études initiales qui constituent un point de repère de cette étude.

\subsection{Procédure}

La tâche de compréhension se déroule sur un des lieux de vie des participants: famille, association, école, collège ou université. Elle se déroule dans une pièce isolée en présence du participant, d'un enquêteur et d'un traducteur. Les paroles de l'enquêteur sont uniquement traduites au moment de la présentation de la tâche et du questionnaire sociolinguistique.

Une séance d'entraînement initial permet de s'assurer que le lexique des affirmations (bonbon, boîte rouge, boîte bleue) est compris par l'apprenant. Le nom bonbon étant phonétiquement proche du français en arabe syrien, il est facilement compris par les participants, de même que les adjectifs rouge et bleu. Puis les sujets ont une phase d'entraînement dans laquelle ils entendent plusieurs paires de phrases (il est/ il n'est pas). $\mathrm{Au}$ bout de trois essais réussis d'affilée, l'expérimentateur considère que le principe du jeu est compris et passe à la phase de test.

Chaque paire d'affirmation est écoutée une seule fois. Pour donner sa réponse, le participant dit la couleur de la boîte ou la montre, l'expérimentateur met de côté la boîte désignée en encourageant ( $o k$, bien) mais sans évaluer la réponse. Le rythme de la passation n'est pas imposé, l'enquêteur respecte le temps nécessaire à l'apprenant pour fournir sa réponse.

A la fin, un score est établi pour chaque série (maxi 12) et chaque participant (maxi 36) à partir d'une analyse des boîtes mises de côté.

\section{Les résultats}

Nous présentons d'abord les résultats globaux puis par série et contraste puis enfin les scores individuels en fonction de la durée d'exposition intensive afin de répondre aux questions suivantes:

- Y a-t-il un effet de l'âge de première exposition sur la compréhension globale des expressions épistémiques ou sur la compréhension de certaines expressions épistémiques, voire de certains contrastes épistémiques et si oui, lesquels?

- La relation entre scores individuels au test de compréhension et durée d'exposition intensive au français varie-t-elle selon l'âge?

\subsection{Résultats globaux}

Les résultats globaux au test de compréhension des expressions épistémiques montrent que le score moyen des adultes, 25.73, est plus élevé que celui des adolescents et des enfants respectivement 22.33 et 21.33 (Tableau 4). Un test d'ANOVA à un facteur montre toutefois que cette différence n'est pas significative.

Tableau 4. Score moyen et écart-type des adultes, adolescents et enfants au test de compréhension des expressions modales épistémiques, trois séries confondues (maxi $=36)$

\begin{tabular}{llll}
\hline & $\begin{array}{l}\text { Adultes }(18+) \\
\mathrm{n}=15\end{array}$ & $\begin{array}{l}\text { Adolescents (11-17) } \\
\mathrm{n}=12\end{array}$ & $\begin{array}{l}\text { Enfants (6-10) } \\
\mathrm{n}=12\end{array}$ \\
\hline $\mathrm{M}$ & 25.73 & 22.33 & 21.33 \\
\hline
\end{tabular}




$\begin{array}{llll}\text { ET } & 5.84 & 5.66 & 5.89\end{array}$

\subsection{Résultats par série et contraste épistémique}

L'analyse des scores par série ou type d'encodage (A adverbe, B verbe modal et C verbe cognitif) confirme l'avantage du groupe adulte sur les deux autres groupes, toutes séries confondues. Les scores des adolescents sont supérieurs à ceux des enfants pour les séries "adverbe" et "verbe cognitif". Pour la série "verbe modal", les enfants réussissent légèrement mieux que les adolescents, 6.91 contre 6.66 , mais ce score reste proche du hasard (maximum 12).

Tableau 5. Score moyen (écart-type) par groupe d'âge selon le type d'encodage de l'épistémicité $(\operatorname{maxi}=12)$

\begin{tabular}{llll}
\hline & $\begin{array}{l}\text { Adultes }(18+) \\
\mathrm{n}=15\end{array}$ & $\begin{array}{l}\text { Adolescents (11-17) } \\
\mathrm{n}=12\end{array}$ & $\begin{array}{l}\text { Enfants (6-10) } \\
\mathrm{n}=12\end{array}$ \\
\hline Adv & $8.73(2.5)$ & $7.41(2.7)$ & $6.66(2.5)$ \\
\hline V mod. & $8.2(2.1)$ & $6.66(1.8)$ & $6.91(2.3)$ \\
\hline V cog. & $8.8(2.1)$ & $8.08(2.4)$ & $7.75(2.7)$ \\
\hline
\end{tabular}

Un test d'analyse de variance avec les variables "type d'encodage" et "groupe d'âge" comme variables intra-individuelles montre un effet du groupe d'âge de première exposition intensive sur le résultat, $\mathrm{F}(2,108)=4.3586, \mathrm{p}<0.05$, mais aucun effet du type d'encodage et aucun effet d'interaction groupe d'âge $x$ type d'encodage.

Les analyses de l'effet du groupe sur la compréhension d'un type d'encodage de l'épistémicité attestent d'un effet de groupe pour les seules expressions adverbiales de l'épistémicité. L'analyse de variance à deux facteurs (groupe d'âge et contraste épistémique) atteste d'un effet de groupe significatif $\mathrm{F}(2,108)=4.1114, \mathrm{p}<0.05$ ainsi que d'un effet significatif de contraste, $\mathrm{F}(2,108)=4.3459, \mathrm{p}<0.05$, mais pas d'effet d'interaction. La figure 1 ci-dessous illustre le fait que les adultes ont des scores supérieurs aux enfants et aux adolescents lorsque la modalité épistémique est encodée au moyen d'un adverbe. L'écart entre le score adulte moyen et les scores enfants et adolescents moyens est particulièrement important pour le contraste bien sûr/probablement.

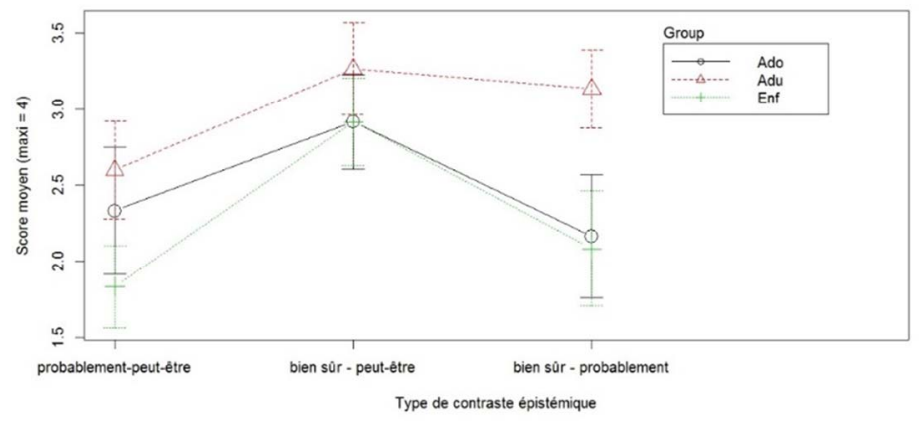

Figure 1. Score moyen des expressions épistémiques adverbiales par groupe d'âge selon le type de contraste 
Pour la série "verbe modal”, il n'y a pas d'effet significatif de la variable groupe d'âge. Cependant, nous observons dans la figure 2 ci-dessous que le score adulte moyen pour le contraste est/doit être est bien supérieur au score des enfants et des adolescents. Une analyse de variance à un facteur montre de fait un effet significatif du groupe d'âge $\mathrm{F}$ (2, 36) $=5.3383, \mathrm{p}<0.01$.

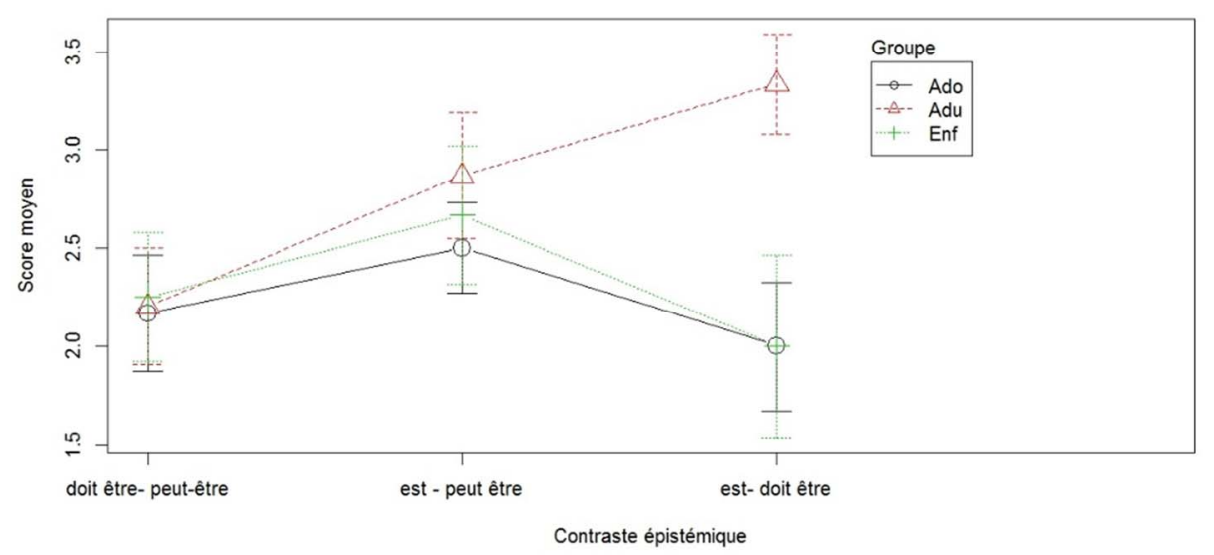

Figure 2. Score moyen de compréhension des contrastes épistémiques verbaux par groupe d'âge, selon le type de contraste

\subsection{Scores individuels et durée d'exposition intensive}

Enfin, la figure 3 ci-dessous présente sous forme de trois nuages de points la dispersion des scores individuels des enfants, adolescents et adultes en fonction de leur durée d'exposition intensive au français. Dans les trois groupes, lorsque la durée d'exposition intensive est inférieure à trois mois, le score obtenu relève de la chance (autour de 18 points sur 36 ). Dans le groupe adulte, à partir du $5^{\text {ème }}$ mois d'immersion, les individus ont tous des scores supérieurs à 27 points sur 36. Ce n'est pas le cas dans les groupes adolescent et enfant dans lesquels on observe des scores individuels relevant du hasard à 8 , 9 et 11 mois d'immersion.

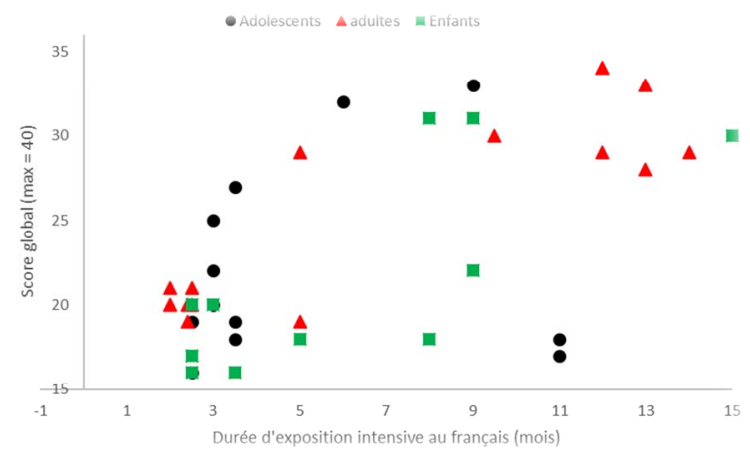

Figure 3. Scores individuels de compréhension selon la durée d'exposition intensive au français 
En d'autres termes, au-delà de 5 mois d'exposition intensive au français, les apprenants enfants ou adolescents présentent des scores variables alors que les adultes présentent tous des scores élevés.

\section{Discussion et conclusion}

L'objectif de cette étude est de savoir si l'âge de première exposition intensive au français a un impact sur la compréhension des expressions épistémiques dans les 15 premiers mois. De nombreuses études ont montré un avantage de l'arrivée précoce dans le pays d'immigration plusieurs années plus tard mais très peu d'études ont documenté les parcours acquisitionnels de locuteurs d'âge distincts dans les premiers stades d'acquisition de la L2. Et aucune étude à notre connaissance ne s'est intéressée à la compréhension du français L2 à ce stade, qui plus est de la compréhension des expressions épistémiques.

Les résultats montrent que dans les 15 premiers mois d'exposition intensive au français les adultes comprennent les contrastes épistémiques en moyenne mieux que les adolescents et les enfants. En particulier, les adultes comprennent nettement mieux que les adolescents et les enfants la différence la différence entre est et doit être. Ceci est surtout valable à partir du $6^{\text {ème }}$ mois d'exposition intensive. Avant, les scores attestent dans tous les groupes d'âge de résultats dus à des réponses au hasard. A partir du $6^{\text {ème }}$ mois d'exposition intensive, tous les participants de plus de 18 ans ont des scores élevés alors que cela ne concerne que quelques membres des groupes enfant et adolescent. L'âge semble ainsi conférer aux adultes un avantage pour comprendre les expressions épistémiques alors que pour les enfants et les adolescents, l'âge seul ne permet pas de prédire le score au test. D'autres facteurs, comme la quantité et la qualité de l'input reçu, sont susceptibles d'expliquer les performances des enfants, comme le soulignent Ågren et al. (2014) ou Granfeldt (2016). Il est aussi intéressant de souligner que la préférence des enfants pour les moyens grammaticaux et celle des adultes pour les moyens lexicaux en production (Schlyter 2003, VanPatten 2004) ne semble pas avoir d'équivalent en compréhension dans cette phase initiale. Ces résultats doivent toutefois être considérés avec prudence en raison de la taille de l'échantillon.

Néanmoins, cette étude ouvre des pistes intéressantes. Par exemple la comparaison des résultats de cette étude de compréhension à ceux d'études de production d'expressions épistémiques menées auprès d'apprenants également arabophones (L1 arabe marocain) suggèrent une asymétrie: les apprenants de notre étude comprennent le contraste sémantique entre des expressions épistémiques (bien sûr/probablement, est/doit être) qu'en production, des apprenants arabophones n'emploient pas après 3 à 4 ans d'exposition intensive. Ce constat invite à explorer davantage les asymétries compréhension/ production en L2 pour découvrir d'autres dimensions du processus acquisitionnel. Il est aussi intéressant d'observer que dans les 15 premiers mois d'exposition à la L2, les enfants ne présentent pas, par rapport aux adultes, un avantage particulier dans le traitement et la compréhension des expressions modales épistémiques. Ce résultat contraste avec l'avantage comparatif des enfants par rapport aux adultes à atteindre un stade dit ultime de développement, maintes fois documenté dans les études antérieures autour de la période critique. Notre étude montre que la variation des observables (compréhension, stade initial, expressions épistémiques d'émergence tardive en L1) laisse entrevoir des effets de l'âge plus complexes et divers qu'il n'y paraît. L'étude apporte ainsi des données qui vont dans le sens de l'intuition, peu développée par la suite, de Krashen, Long et Scarcella (1979). Elle apporte aussi quelques éléments en faveur du deuxième scénario développemental (S2) esquissé en introduction : si en langue seconde, les adultes comprennent plus vite que les enfants la valeur d'épistémicité de certaines formes linguistiques, lexicales ou 
grammaticales, alors on pourrait penser qu'ils atteignent aussi plus rapidement un point de fossilisation alors que les enfants disposeraient d'une marge de progression plus large.

Cette étude n'a bénéficié d'aucun financement extérieur mais de dons inestimables. Nous tenons à remercier pour leur confiance et leur générosité toutes celles et ceux qui ont donné leur langue, ainsi que les enseignantes, animatrices, amies et parents qui nous ont guidées jusqu'à elles et eux.

\section{Références bibliographiques}

Abrahamsson, N., \& Hyltenstam, K. (2009). Age of onset and nativelikeness in a second language: Listener perception versus linguistic scrutiny. Language learning, 59(2), 249-306.

Ågren, M., Granfeldt, J., \& Thomas, A. (2014). Combined effects of age of onset and input on the development of different grammatical structures: A study of simultaneous and successive bilingual acquisition of French. Linguistic Approaches to Bilingualism, 4(4), 462-493.

Bassano, D., Hickmann, M., Champaud, C. (1992). Epistemic modality in French children's discourse: "to be sure" or "not to be sure"? Journal of Child Language, 19, 389-413.

Bassano, D. \& Mendes-Maillochon, I. (1994). Early grammatical and prosodic marking of utterance modality in French: a longitudinal case study, Journal of Child Language, 21, 649-675.

Bassano, D. (1996). Functional and formal constraints on the emergence of epistemic modality: A longitudinal study on French. First Language, 16(46), 077-113.

Bialystok, E., \& Hakuta, K. (1994). In other words The science and psychology of second-language acquisition. New York: Basic Books.

Birdsong, D. (2005). Nativelikeness and non-nativelikeness in L2A research. IRAL - International Review of Applied Linguistics in Language Teaching, 43(4), 319-328.

Birdsong, D., \& Molis, M. (2001). On the Evidence for Maturational Constraints in Second-Language Acquisition. Journal of Memory and Language, 44(2), 235-249.

Byrnes, J. P., \& Duff, M. A. (1989). Young children's comprehension of modal expressions. Cognitive Development, 4(4), 369-387.

Choi, S. (2005). Acquisition of modality. In Frawley W. (Ed), The expression of modality (pp. 141-171). Berlin: De Gruyter.

DeKeyser, R. (2003) Implicit and explicit learning. Dans Catherine J. Doughty \& Michael H. Long (coords) The Handbook of Second Language Acquisition, (pp. 313-348). Oxford, Blackwell.

Dimroth, C. (2008). Age Effects on the Process of L2 Acquisition? Evidence From the Acquisition of Negation and Finiteness in L2 German. Language Learning, 58(1), 117-150.

Dimroth, C. (2009). L'acquisition de la finitude en allemand L2 à différents âges. Acquisition et interaction en langue étrangère, (Aile... Lia 1), 39-61.

Dimroth, C., \& Haberzettl, S. (2012). The older the better, or more is more: language acquisition in childhood. In Watorek, M., Benazzo, S. \& Hickmann, M. (Eds.). Comparative perspectives on language acquisition: A tribute to Clive Perdue. Bristol: Multilingual Matters. 324-349.

Gaonac'h, D. (2006). L'apprentissage précoce d'une langue étrangère : le point de vue de la psycholinguistique. Paris: Hachette.

Granena, G., \& Long, M. H. (2013). Age of onset, length of residence, language aptitude, and ultimate L2 attainment in three linguistic domains. Second Language Research, 29(3), 311-343.

Granfeldt, J. (2016). Rôles de l'âge, de l'input et de la L1 dans le développement du français par des enfants L2. Revue française de linguistique appliquée, 21(2), 33-48.

Hirst, W., \& Weil, J. (1982). Acquisition of epistemic and deontic meaning of modals. Journal of Child Language, 9(03). https://doi.org/10.1017/S0305000900004967

Hopp, H. (2010). Ultimate attainment in L2 inflection: Performance similarities between non-native and native speakers. Lingua, 120(4), 901-931.

Johnson, J.S. \& Newport, E.L. (1989). Critical period effects in second language learning: The influence of maturational state on the acquisition of English as a second language. Cognitive Psychology. 21.60-99. 
Hyltenstam, K., \& Abrahamsson, N. (2003). Age of onset and ultimate attainment in near-native speakers of Swedish as a second language. In Fraurund K. \& Hyltenstam K. (eds), Multilingualism in Global and Local Perspectives, (p. 319-340), Center for Research on Bilingualism: Stockholm.

Kihlstedt, M. (2015). Acquisition of the imparfait in L2 French in adults and children: The same or different?: A comparative case study of Swedish adults and children in an immersion setting. Language, Interaction and Acquisition, 6(1), 74-106.

Köpke, B. (2004). Neurolinguistic aspects of attrition, Journal of Neurolinguistics, 17 (1), 3-30.

Krashen, S. D., Long, M. A., \& Scarcella, R. C. (1979). Age, Rate and Eventual Attainment in Second Language Acquisition. TESOL Quarterly, 13(4), 573-582.

Kupisch, T., D. Barton, T. Lein, J. Schröder, I. Stangen \& A. Stöhr. 2014. Acquisition outcomes across domain in adult heritage speakers of French. Journal of French Language Studies 24(3). 347-376.

Lenneberg, E. H. (1967). Biological Foundations of Language. New York, NY: Wiley.

Meisel, J. (2011). First and Second Language Acquisition: Parallels and Differences. Cambridge: Cambridge University Press.

Mifka-Profozic N. (2017). Processing epistemic modality in a second language: A self paced reading study. International Review of Applied Linguistics and Language Teaching 55(3), 245-264.

Mitchell, R. \& Myles, F. (2004). Second language learning theories. Second edition. London: Hodder Arnold.

Moore, C., Pure, K. \& Furrow, D. (1990). Children's understanding of the modal expression of speaker certainty and uncertainty and its relation to the development of a representational theory of mind. Child Development 61(3), 722-730.

Newport, E.L. (1990). Maturational Constraints on Language Learning. Cognitive Science 14 (1): 1128.

Noveck, I. A., Ho, S., \& Sera, M. (1996). Children's understanding of epistemic modals. Journal of Child Language, 23(03), 621-643.

Schlyter S. (2003). Development of finiteness in children and adults acquiring French. In Dimroth C. \&Starren M. (eds), Studies in Bilingualism 26, 15-44.

Schimke, S., \& Dimroth, C. (2017). The influence of finiteness and lightness on verb placement in L2 German: Comparing child and adult learners. Second Language Research,

Schmidt, M. (2014)._. The debate on maturational constraints in bilingual development: a perspective from first language attrition. Language acquisition 21(4), 386-410.

Singleton, D. M., \& Ryan, L. (2004). Language Acquisition: The Age Factor. Bristol: Multilingual Matters.

Stephany, U. (1995). Function and Form of Modality in First and Second Language Acquisition. In From pragmatics to syntax: modality in second language acquisition (Anna Giacalone Ramat/ Grazia Crocco Galèas, Vol. 405, p. 105-120). Tübingen: Günter Narr Verlag.

Ünal, E., \& Papafragou, A. (2016). Production-comprehension asymmetries and the acquisition of evidential morphology. Journal of Memory and Language, 89, 179-199.

Vanhove M., Miller C. \& Caubet D. (2009). The grammaticalization of modal auxiliaries in Maltese and Arabic vernaculars of the Mediterranean area. In Van der Auwera J. (Ed.), Grammaticalization of modal particles, (pp. 325-362), New York : Mouton de Gruyter.

Véronique, D. (1995). Acquisition des modalités en français langue étrangère et développement des modalités dans les créoles français. In Giacalone Ramat A \& Grazia Crocco G. (eds), From pragmatcis to syntax: modality in second language acquisition (p. 59-82). Tübingen: Günter Narr Verlag.

Véronique, D., Stoffel, H., \& Giacomi, A. (2000). Modalisation et modalités. In Appropriation du français par des Marocains arabophones à Marseille: bilan d'une recherche. Aix-en-Provence: Publication de l'Université de Provence.

\section{Annexe}

Annexe 1. Le questionnaire sociolinguistique

L'enquêteur pose les questions en français, le traducteur les traduit en arabe, puis traduit la réponse de l'apprenant en français.

Annexe 1.1. Version enfants et adolescents 
Sexe: Âge :

Pays de naissance :

Date d'arrivée en France :

Nom école/ collège/ lycée en France :

Classe fréquentée :

Langues parlées à la maison en Syrie:

Âge de première scolarisation en Syrie :

Langue de scolarisation :

Langues étrangères apprises (âge de début d'apprentissage- âge de fin d'apprentissage) à l'école et en dehors:

Activités en dehors de la classe en France et langues parlées :

Annexe 1.2. Version adultes

Sexe: Âge : ..........Pays de naissance :

Date d'arrivée en France :

Langues entendues et parlées enfant à la maison:

Âge de première scolarisation en Syrie :

Langue de scolarisation :

Langues étrangères apprises (âge de début d'apprentissage- âge de fin d'apprentissage) :

Part du contenu compris dans un film

- en anglais :

- en français :

Qualification en Syrie :

Professions exercées en Syrie :

Cours de français suivis en France (en ligne vs en classe, lieu, niveau, nombre d'heures) :

Type de logement (résidence étudiante, colocation, appartement familial, etc.) :

Activités en dehors de la classe et langues parlées :

Annexe 1.3 Extraits des données enfants et adolescents

\begin{tabular}{|l|l|l|l|l|l|}
\hline Id & Genre & âge & $\begin{array}{l}\text { Exp.int. } \\
\text { (mois) }\end{array}$ & Classe & Scolarité et langues \\
\hline Enf 2 & $\mathrm{M}$ & 8 & 3 & $\mathrm{CE} 2$ & $\begin{array}{l}\text { 6-8 ans : école primaire, Syrie, arabe et anglais } \\
\text { (notions) }\end{array}$ \\
\hline Enf 8 & $\mathrm{F}$ & 8 & 3,5 & $\mathrm{CE} 1$ & $\begin{array}{l}\text { 6-7 ans : école primaire, Jordanie, arabe et } \\
\text { anglais (notions) }\end{array}$ \\
\hline Ado 3 & $\mathrm{F}$ & 15 & 3 & $3^{\text {eme }}$ & $\begin{array}{l}\text { 6-12 ans : école primaire, Syrie, arabe et anglais } \\
\text { (notions), 12-15 secondaire, Turquie, turc }\end{array}$ \\
\hline Ado 9 & $\mathrm{F}$ & 13 & 11 & $6^{\text {eme }}$ & $\begin{array}{l}\text { 3-7 ans : maternelle et } 1^{\text {ere }} \text { année primaire, } \\
\text { Syrie, arabe } ; 7-12, \text { primaire, Liban, arabe et } \\
\text { anglais }\end{array}$ \\
\hline
\end{tabular}

Annexe 1.4 Extraits des données adultes

\begin{tabular}{|l|l|l|l|l|l|}
\hline Id & Genre & $\hat{\text { Age }}$ & $\begin{array}{l}\text { Exp. int. } \\
\text { (mois) }\end{array}$ & Qualification et langues & français \\
\hline Adu 8 & M & 19 & 12 & $\begin{array}{l}\text { Interruption du secondaire à 13 ans, } \\
\text { anglais (8-13), français (12-13), turc } \\
\text { (Turquie, 7 mois) }\end{array}$ & $\begin{array}{l}\text { Cours FLE } \\
\text { intensifs, } \\
\text { université }\end{array}$ \\
\hline Adu 11 & M & 30 & 9,5 & $\begin{array}{l}\text { Licence d'arabe à l'université en } \\
\text { Syrie, anglais (6-20), russe (Russie 1 } \\
\text { an), turc (Turquie, 3 ans), comprend } \\
\text { le kurde }\end{array}$ & $\begin{array}{l}\text { Cours FLE } \\
\text { intensifs, } \\
\text { université }\end{array}$ \\
\hline
\end{tabular}

